Published in final edited form as:

Neuroimage. 2015 February 1; 106: 441-450. doi:10.1016/j.neuroimage.2014.10.061.

\title{
Rapid $T_{2}$ - and susceptometry-based $\mathrm{CMRO}_{2}$ quantification with interleaved TRUST (iTRUST)
}

\author{
Zachary B. Rodgers, Erin K. Englund, Michael C. Langham, Jeremy F. Magland, and Felix \\ W. Wehrli* \\ Laboratory for Structural NMR Imaging, Department of Radiology, University of Pennsylvania \\ Medical Center, Philadelphia, PA, USA
}

\section{Abstract}

Susceptometry-based oximetry (SBO) and $T_{2}$-relaxation-under-spin-tagging (TRUST) are two promising methods for quantifying the cerebral metabolic rate of oxygen $\left(\mathrm{CMRO}_{2}\right)$, a critical parameter of brain function. We present a combined method, interleaved TRUST (iTRUST), which achieves rapid, simultaneous quantification of both susceptometry- and $T_{2}$-based $\mathrm{CMRO}_{2}$ via insertion of a flow-encoded, dual-echo gradient-recalled echo (OxFlow) module within the $T_{1}$ recovery portion of the TRUST sequence. In addition to allowing direct comparison between SBO- and TRUST-derived venous oxygen saturation $\left(Y_{\mathrm{v}}\right)$ values, iTRUST substantially improves TRUST temporal resolution for $\mathrm{CMRO}_{2}$ quantification and obviates the need for a separate blood flow measurement following TRUST acquisition. iTRUST was compared directly to TRUST and OxFlow alone in three resting subjects at baseline, exhibiting close agreement with the separate techniques and comparable precision. These baseline data as well as simulation results support the use of two instead of the traditional four $T_{2}$ preparation times for $T_{2}$ fitting, allowing simultaneous quantification of susceptometry- and $T_{2}$-based $Y_{\mathrm{v}}\left(\right.$ and $\left.\mathrm{CMRO}_{2}\right)$ with three- and six-second temporal resolution, respectively. In 10 young healthy subjects, iTRUST was applied during a 5\% $\mathrm{CO}_{2}$ gas mixture-breathing paradigm. $T_{2}$-based $Y_{\mathrm{v}}$ values were lower at baseline relative to susceptometry $\left(62.3 \pm 3.1\right.$ vs. $\left.66.7 \pm 5.1 \% \mathrm{HbO}_{2}, P<0.05\right)$, but increased more in response to hypercapnia. As a result, $T_{2}$-based $\mathrm{CMRO}_{2}$ decreased from $140.4 \pm 9.7$ to $120.0 \pm 9.5 \mu \mathrm{Mol} / 100 \mathrm{~g} /$ min, a significant $-14.6 \pm 3.6 \%$ response $(P<0.0001)$, whereas susceptometry-based $\mathrm{CMRO}_{2}$ changed insignificantly from $123.4 \pm 18.7$ to $127.9 \pm 25.7$, a $3.3 \pm 9.7 \%$ response $(P=0.31)$. These differing results are in accord with previous studies applying the parent OxFlow or TRUST sequences individually, thus supporting the reliability of iTRUST but also strongly suggesting that a systematic bias exists between the susceptometry- and $T_{2}$-based $Y_{\mathrm{v}}$ quantification techniques.

\section{Keywords}

$\mathrm{CMRO}_{2}$; Blood oxygen saturation; TRUST; MR susceptometry; Phase-contrast MRI; Hypercapnia

\footnotetext{
"Corresponding author at: Laboratory for Structural NMR Imaging, Department of Radiology, University of Pennsylvania Medical Center, 1 Founders Building, MRI Education Center, 3400 Spruce Street, Philadelphia, PA 19104, USA. Fax: +1 2156627263. wehrli@mail.med.upenn.edu (F.W. Wehrli).
} 


\section{Introduction}

The human brain comprises only $2 \%$ of total body mass, but accounts for approximately $20 \%$ of total body oxygen consumption (Rolfe and Brown, 1997). Because the brain is almost entirely dependent on aerobic metabolism to meet its energetic demands, irreversible ischemic damage will result in minutes if oxygen delivery is disrupted. Unlike surrogate markers of metabolism such as cerebral blood flow (CBF) or blood-oxygen-level dependent (BOLD) functional MRI (fMRI) signal, the cerebral metabolic rate of oxygen $\left(\mathrm{CMRO}_{2}\right)$ provides a direct measure of brain oxygen consumption. $\mathrm{CMRO}_{2}$ changes significantly over the course of neonatal development (Liu et al., 2014a) and aging (Peng et al., 2014), and is altered in many of the most common neurologic diseases, including mild cognitive impairment (Liu et al., 2014b) and Alzheimer's disease (Ishii et al., 1996), Parkinson's disease (Borghammer et al., 2010), and multiple sclerosis (Ge et al., 2012). However, $\mathrm{CMRO}_{2}$ is relatively stable across healthy subjects at baseline (Xu et al., 2009; Jain et al., 2010), and in response to physiologic challenges such as hypercapnia (Chen and Pike, 2010; Xu et al., 2011; Jain et al., 2011), hypoxia (Xu et al., 2012a), and apnea (Rodgers et al., 2013a). Thus, $\mathrm{CMRO}_{2}$ is an important quantity for understanding brain function in health and disease.

The gold standard for $\mathrm{CMRO}_{2}$ quantification is triple-oxygen positron emission tomography (PET) imaging (Ito et al., 2005), yet the technique is rarely applied in humans due to the radiation exposure and complexity of the protocol. Moreover, long scan times restrict PET to measuring resting-state $\mathrm{CMRO}_{2}$. MRI provides a non-invasive, non-contrast alternative. During the past two decades, BOLD fMRI has been applied extensively to study neuronal activation in health and disease (Matthews et al., 2006; Kim and Ogawa, 2012). However, BOLD signal does not provide a direct measure of brain oxygen metabolism, but rather reflects a complex interplay between $\mathrm{CBF}$, cerebral blood volume, and tissue properties such blood vessel diameter, in addition to $\mathrm{CMRO}_{2}$ (Blockley et al., 2013).

Recently, a number of MR-based approaches for direct quantification of cerebral venous oxygen saturation $\left(Y_{\mathrm{v}}\right)$ have been proposed (Jain et al., 2010, 2014; Haacke et al., 1997; Fernandez-Seara et al., 2006; Fan et al., 2012, 2014; Wright et al., 1991; Golay et al., 2001; Lu and Ge, 2008; Chen and Pike, 2009; Bolar et al., 2011; Qin et al., 2011; Guo and Wong, 2012; Krishnamurthy et al., 2013; An and Lin, 2000; He and Yablonskiy, 2007; Bulte et al., 2012; Gauthier et al., 2012). In combination with phase-contrast MRI (PC-MRI) or arterial spin labeling (ASL) CBF quantification, these techniques allow for determination of $\mathrm{CMRO}_{2}$ via the Fick Principle (Kety and Schmidt, 1948):

$$
\mathrm{CMRO}_{2}=\mathrm{C}_{\mathrm{a}} \cdot \mathrm{CBF} \cdot\left(Y_{\mathrm{a}}-Y_{\mathrm{v}}\right)
$$

where $\mathrm{C}_{\mathrm{a}}$ is the arterial oxygen content of blood in $\mu \mathrm{Mol} / 100 \mathrm{~mL}$ and $Y_{\mathrm{a}}$ the arterial oxygen saturation in percent hemoglobin oxygen saturation $\left(\% \mathrm{HbO}_{2}\right)$, which can be measured with pulse oximetry. Total CBF (tCBF) is typically reported in units of $\mathrm{mL}$ blood/100 $\mathrm{g}$ brain tissue/minute, giving $\mathrm{CMRO}_{2}$ in units of $\mu \mathrm{Mol} / 100 \mathrm{~g} /$ minute. 
Measurement of $Y_{\mathrm{v}}$ poses the most significant technical challenge in $\mathrm{CMRO}_{2}$ determination. Techniques for $Y_{\mathrm{v}}$ quantification can be categorized based on the contrast mechanism venous blood magnetic susceptibility (Jain et al., 2010; Haacke et al., 1997; FernandezSeara et al., 2006; Fan et al., 2012, 2014), $T_{2}$ (Wright et al., 1991; Golay et al., 2001; Lu and Ge, 2008; Chen and Pike, 2009; Bolar et al., 2011; Qin et al., 2011; Guo and Wong, 2012; Jain et al., 2014; Krishnamurthy et al., 2013), $T_{2}{ }^{\prime}$ (An and Lin, 2000; He and Yablonskiy, 2007), or $T_{2}{ }^{*}$ (BOLD) (Bulte et al., 2012; Gauthier et al., 2012) - as well as spatial specificity - large-vessel/whole-brain (Jain et al., 2010, 2014; Haacke et al., 1997; Fernandez-Seara et al., 2006; Fan et al., 2012; Wright et al., 1991; Golay et al., 2001; Lu and Ge, 2008; Chen and Pike, 2009; Qin et al., 2011), small-vessel/regional (Fan et al., 2014; Krishnamurthy et al., 2013), or parenchymal/voxel-wise (Bolar et al., 2011; Guo and Wong, 2012; An and Lin, 2000; He and Yablonskiy, 2007; Bulte et al., 2012; Gauthier et al., 2012). Regional and voxel-wise approaches are clearly desirable due to the heterogeneous nature of brain functional activation and pathology. However, these techniques have scan times on the order of several minutes, precluding dynamic measurements, and tend to suffer from low signal-to-noise ratio (SNR), requiring significant spatial averaging to achieve acceptable precision and thus negating the utility of regional or voxel-wise measurement. In comparison, techniques for whole-brain $Y_{\mathrm{v}}$ quantification are fast, robust, and easy to implement.

The two best-established methods for global $Y_{\mathrm{v}}$ quantification are $T_{2}$-relaxation-under-spintagging (TRUST) (Lu and Ge, 2008) and susceptometry-based oximetry (SBO) (Jain et al., 2010). Both methods involve quantification of intravascular $Y_{\mathrm{v}}$ in the superior sagittal sinus (SSS), the largest cerebral venous drainage vessel, which, in combination with PC-MRI quantification of tCBF, can be used to determine $\mathrm{CMRO}_{2}$ via Eq. (1). In the case of TRUST, tCBF measurement requires a separate PC-MRI acquisition (Xu et al., 2009). However, because PC-MRI and SBO are both gradient-recalled echo (GRE) sequences, they can be naturally combined into a single sequence, which we term OxFlow. This hybrid sequence was originally implemented via a two-slice interleaved approach with $\mathrm{CMRO}_{2}$ quantification temporal resolution of 25 seconds (Jain et al., 2010). Recently, addition of view-sharing and SSS-based estimation of tCBF improved OxFlow temporal resolution to three seconds, allowing study of the regulation of $\mathrm{CMRO}_{2}$ in response to dynamic physiologic paradigms such as breath-hold apnea (Rodgers et al., 2013a). Compared to OxFlow, TRUST has inherently lower temporal resolution, compounded by the need for a separate PC-MRI measurement to quantify $\mathrm{CMRO}_{2}$. Furthermore, the relationship between $T_{2}, Y_{\mathrm{v}}$, and hematocrit (Hct) is non-linear, and must be calibrated to both pulse sequence parameters and field strength. However, unlike SBO, TRUST is vessel geometry independent, less sensitive to partial volume effects, and does not require background phase removal.

A particularly important application of $\mathrm{CMRO}_{2}$ quantification is investigating the metabolic response to hypercapnia. Hypercapnia is relevant to a number of common diseases, including asthma, chronic obstructive pulmonary disease, obstructive sleep apnea, and congestive heart failure. Furthermore, knowledge of the $\mathrm{CMRO}_{2}$ response to hypercapnia is of substantial importance to functional imaging, where hypercapnia is routinely used for 
'calibrating' the fMRI signal (Davis et al., 1998), often under the assumption that hypercapnia is isometabolic (i.e., does not affect $\mathrm{CMRO}_{2}$ ). However, the $\mathrm{CMRO}_{2}$ response to a hypercapnic stimulus remains controversial (Yablonskiy, 2011), with previous studies reporting a wide range of results from reduced, to unchanged, to increased $\mathrm{CMRO}_{2}$. An early MRI study using $T_{2}$-based $Y_{\mathrm{v}}$ quantification reported an isometabolic response (Chen and Pike, 2010); however, $\mathrm{CMRO}_{2}$ responses to mild and moderate hypercapnia were in different directions (5.0\% and $-6.8 \%$, respectively) and based on a calibration plot derived from room temperature blood samples (Chen and Pike, 2009), potentially impacting the accuracy of in vivo $T_{2}$ quantification (Spees et al., 2001). Subsequently, both OxFlow and TRUST have been applied to study the $\mathrm{CMRO}_{2}$ response to hypercapnia using similar cohorts and experimental protocols involving a $5 \% \mathrm{CO}_{2}$ gas mixture delivery (Xu et al., 2011; Jain et al., 2011). While OxFlow data supported an isometabolic $\mathrm{CO}_{2}$ response, the TRUST study found a significant $13.4 \pm 2.3 \%$ (mean \pm standard error, $N=14$ ) decrease in $\mathrm{CMRO}_{2}$. This discrepancy is disconcerting given both the importance of understanding the $\mathrm{CMRO}_{2}$ response to hypercapnia as well as the increasing application of TRUST and OxFlow in studying $\mathrm{CMRO}_{2}$ responses to other stimuli and disease states. A recent study directly comparing resting TRUST- and SBO-derived $Y_{\mathrm{v}}$ values in the same cohort (Barhoum et al., 2014) found TRUST $Y_{\mathrm{v}}$ values to be slightly lower (mean \pm SD of $63.2 \pm$ 4.1 vs. $65.9 \pm 3.3 \% \mathrm{HbO}_{2}, P<0.01$ ). However, this baseline difference does not by itself explain the discrepancy in the hypercapnia results, which depends on the relative change in $Y_{\mathrm{v}}$ in response to the stimulus.

In this work, we propose a combined technique - termed interleaved TRUST (iTRUST) whereby an OxFlow module is interleaved within the $T_{1}$ recovery period of the TRUST sequence. This approach has two distinct benefits. First, it obviates the need for separate, non-simultaneous measurement of tCBF following the TRUST acquisition, substantially improving TRUST temporal resolution for $\mathrm{CMRO}_{2}$ quantification. Second, it allows for direct comparison of $Y_{\mathrm{v}}$ quantified via magnetic susceptibility and $T_{2}$ measurement of blood. Further temporal acceleration compared to TRUST is achieved by using fewer tag-control image pairs for $T_{2}$ fitting. Both the combination of the techniques as well as the use of fewer $T_{2}$ fitting points are validated in simulations and in vivo. The sensitivity of the technique to detect dynamic changes is demonstrated in response to breath-hold apnea. Finally, iTRUST is applied in a cohort of young healthy subjects during a $\mathrm{CO}_{2}$ gas mixture-breathing paradigm with the goal of further investigating the potential disagreement between the TRUST and OxFlow techniques with regard to the hypercapnic $\mathrm{CMRO}_{2}$ response.

\section{Theory}

\section{Susceptometry-based quantification of $Y_{v}(S B O)$}

Susceptometry-based oximetry exploits the relative paramagnetism of deoxygenated versus oxygenated hemoglobin, which causes the susceptibility of blood relative to surrounding tissue, $\Delta \chi$, to be linearly related to venous oxygen saturation:

$$
\Delta \chi=\operatorname{Hct} \cdot\left(\Delta \chi_{\mathrm{do}}\left(1-Y_{\mathrm{v}}\right)+\Delta \chi_{\mathrm{oxy}}\right)
$$


where $\Delta \chi_{\text {do }}$ and $\Delta \chi_{\text {oxy }}$ are the experimentally determined volume susceptibility differences between fully oxygenated and deoxygenated erythrocytes and fully oxygenated erythrocytes and water, respectively. Values of $4 \pi \times 0.273$ and $4 \pi \times 0.008$ p.p.m. (SI units) are used for $\Delta \chi_{\mathrm{do}}$ and $\Delta \chi_{\mathrm{oxy}}$, based on ex vivo calibration experiments (Spees et al., 2001; Jain et al., 2012).

Blood susceptibility induces a local field offset, $\Delta B$, which can be measured with a field mapping sequence as:

$$
\Delta B=\Delta \phi / \gamma \Delta T E
$$

where $\Delta \varphi$ is the difference in phase accrual between echoes spaced apart by $\Delta T E$ in the blood versus surrounding reference tissue. By modeling the vessel of interest as an infinitely long, circular cylinder, the relationship between $\Delta B$ and $\Delta \chi$ can be calculated analytically:

$$
\Delta B=\frac{1}{6} \Delta \chi B_{0}\left(3 \cos ^{2} \theta-1\right)
$$

where $\theta$ is the vessel angle with respect to the main magnetic field, $\mathrm{B}_{0}$. Combining Eqs. (2)(4) allows for determination of $Y_{\mathrm{v}}$ by measurement of $\Delta \varphi$.

The SSS, the largest cerebral venous drainage vessel, is relatively long and straight when the subject is lying supine in the scanner, and can therefore be effectively approximated by the infinite cylinder model, despite its non-circular cross-section (Jain et al., 2010; Li et al., 2012). The SSS has also been shown to have a $Y_{\mathrm{v}}$ nearly identical to that in the internal jugular vein (Xu et al., 2009; Jain et al., 2014), making it an excellent surrogate for global venous $Y_{\mathrm{v}}$. Furthermore, while field mapping of the internal jugular vein is complicated by the presence of trachea-induced susceptibility artifacts, the field local to the SSS is relatively homogenous.

\section{Combination of SBO and PC-MRI for $\mathrm{CMRO}_{2}$ quantification (OxFlow)}

SBO can be combined with PC-MRI blood flow quantification to allow simultaneous measurement of $Y_{\mathrm{v}}, \mathrm{tCBF}$, and, therefore, $\mathrm{CMRO}_{2}$, from a single sequence. By adding flow encoding to the same dual-echo GRE used for $Y_{\mathrm{v}}$ quantification, SSS blood flow (SSSBF) and $Y_{\mathrm{v}}$ can be quantified from data acquired in the same TR period. SSSBF can then be retrospectively up-scaled to tCBF based on a single measurement of the SSSBF:tCBF ratio at baseline (Rodgers et al., 2013a).

In this study, OxFlow was implemented with a BRISK k-space sampling scheme, with onequarter k-space acquired at each time point (Doyle et al., 1995). BRISK provides reduced motion sensitivity compared to previous view-sharing implementations of OxFlow using Keyhole k-space sampling (Rodgers et al., 2013b; Van Vaals et al., 1993). BRISK images were reconstructed by interpolating across time points using the nearest acquired data at each k-space segment, effectively resulting in a sliding window reconstruction with minimum window width of three seconds (inner 1/8th of $\mathrm{k}$-space) and maximum window 
width of 60 seconds (outer 5/8th of k-space). Other OxFlow sequence parameters include: $\mathrm{TR} / \mathrm{TE}_{1} / \mathrm{TE}_{2}=14.2 / 6.5 / 11.5 \mathrm{~ms}, \mathrm{VENC}=40 \mathrm{~cm} / \mathrm{s}$, reconstructed matrix $=192 \times 192$, and resolution $=1.0 \times 1.0 \times 5.0 \mathrm{~mm}$.

\section{TRUST and interleaved TRUST (iTRUST)}

The TRUST pulse sequence uses a non-selective MLEV-16 CPMG $T_{2}$ preparation of varying effective echo time (eTE) - 0, 40, 80, and $160 \mathrm{~ms}$ - following either an $8 \mathrm{~ms}$ adiabatic hyperbolic secant pulse (bandwidth $=2214 \mathrm{~Hz}$, thickness $=100 \mathrm{~mm}$ ) to invert the blood magnetization (tag), or application of an equivalent off-resonance pulse without gradient (control). Similar in principle to ASL, tag-control subtraction of each eTE image pair isolates the venous blood signal. A nonselective $90^{\circ}$ spoiler RF pulse is applied to reset the magnetization before each tag-control module (Xu et al., 2012b). A two-compartment exchange model is used to relate $Y_{\mathrm{v}}$ to $T_{2}$ :

$$
1 / T_{2}=A+B \cdot\left(1-Y_{\mathrm{v}}\right)+C \cdot\left(1-Y_{\mathrm{v}}\right)^{2}
$$

where $A, B$, and $C$ are Hct- and CPMG spacing $\left(t_{\mathrm{CPMG}}\right)$-dependent constants which have been determined from ex vivo blood samples ( $\mathrm{Lu}$ et al., 2012). $T_{2}$ is quantified by monoexponential fitting of SSS tag-control difference signals versus eTE as:

$$
\Delta S=S_{0} e^{\mathrm{eTE} \cdot\left(1 / T_{1}-1 / T_{2}\right)}
$$

where $S_{0}$ is the difference signal at eTE $=0$ and a $T_{1}$ value of 1.613 seconds is assumed for venous blood (Xu et al., 2012b).

The TRUST sequence used in the present work follows that described in recent literature (Xu et al., 2012b), with a TR of three seconds used to provide an optimal tradeoff between scan duration, accuracy, and precision, allowing a single $Y_{\mathrm{v}}$ value to be quantified every 24 seconds. Important differences relative to the published sequence include insertion of a slice-selective saturation pulse $200 \mathrm{~ms}$ before EPI readout (prior to $T_{2}$ preparation) in order to better suppress static tissue signal (Qin et al., 2011; Jain et al., 2014), and use of a flowcompensated EPI readout with TE of $8 \mathrm{~ms}$ (5/8th partial Fourier readout). Flow compensation prevents flow velocity-dependent signal variations between tag and control images, which could lead to errors in the difference signals, especially in situations of rapidly changing flow (Xu et al., 2012b). An alternative approach to avoiding these effects is use of a shorter TE achieved via parallel imaging (Xu et al., 2012b), though this reduces SNR Other TRUST sequence parameters include: $t_{\mathrm{CPMG}}=10 \mathrm{~ms}$, reconstructed matrix $=64$ $\times 64$, and resolution $=3.4 \times 3.4 \times 5.0 \mathrm{~mm}$.

More than half of the duration of the TRUST sequence consists of dead time, required to allow blood signal to undergo sufficient $T_{1}$ recovery following global saturation before the next $T_{2}$ preparation. In iTRUST, this time is utilized to run an OxFlow module at the same location as the TRUST readout slice (Fig. 1), beginning $350 \mathrm{~ms}$ after the saturation in order to capture the tissue signal at approximately its steady-state longitudinal magnetization. Besides the added OxFlow module, iTRUST is otherwise identical to TRUST. 
It is important to note that the RF pulses played out during the OxFlow module only affect spins in the imaging slice, whereas spins relevant to $T_{2}$-quantification are located outside the imaging slice in the labeling slab. Furthermore, because the OxFlow module is run during both tag and control, any effect on spins in the subsequently acquired EPI images used for $T_{2}$ quantification should be identical, and hence removed by tag-control subtraction.

Likewise, the OxFlow GRE acquisition itself is unaffected by the TRUST sequence because it is acquired only after global magnetization reset.

\section{Materials and methods}

\section{Human subject protocols}

All human subject imaging protocols were approved by the University of Pennsylvania's Institutional Review Board, and subjects provided written informed consent prior to participation. Studies were performed on 10 healthy subjects (age $29 \pm 5$ years, range 24-42, six males and four females) using a 3 T Siemens Tim Trio system (Siemens Medical Solutions, Erlangen, Germany) with a 12-channel (validation study and apnea study) or 32channel (hypercapnia study) receive-only head coil. A vendor provided time-of-flight axial localizer scan was used for slice selection, and retrospectively to determine $\theta$ in Eq. (4). Before each OxFlow or iTRUST acquisition, a two-slice interleaved PC-MRI pulse sequence was run at the level of the internal carotid and vertebral arteries in the neck and the SSS in the head in order to determine the subject's SSSBF:tCBF ratio. OxFlow, TRUST, and iTRUST pulse sequences were programmed in SequenceTree (Magland and Wehrli, 2006).

At the end of each scanning session, a 1-mm-isotropic 3D $T_{1}$-weighted MPRAGE (Mugler and Brookeman, 1990) data set was acquired so that tCBF could be normalized per unit brain mass in each subject. Total brain volume was obtained using the BET tool in FSL (Smith, 2002), and converted to mass based on an average brain density of $1.05 \mathrm{~g} / \mathrm{mL}$ (Herscovitch and Raichle, 1985). Total intracranial mass (gray matter, white matter, and cerebrospinal fluid (CSF)) rather than total parenchymal mass (gray matter and white matter) was used for normalization to facilitate comparison of $\mathrm{CMRO}_{2}$ values with prior studies that did the same (Xu et al., 2009; Jain et al., 2010). It has recently been shown that inclusion of CSF volumes inflow normalization may bias toward underestimation of $\mathrm{CMRO}_{2}$ in older individuals (Peng et al., 2014), though this is not a concern in the present study due to the relatively young age of the subjects.

\section{Validation study}

To test whether the combination of OxFlow and TRUST causes a bias in the measurements of either sequence, equivalent OxFlow, TRUST, and iTRUST sequences were run back to back for four minutes each in three subjects (age $29 \pm 3$ years, range 26-34, two males and one female). This protocol corresponds to 10 repetitions of TRUST and iTRUST with 24second temporal resolution, and 80 repetitions of OxFlow with three-second temporal resolution. For the OxFlow only sequence, TR was increased to $31.25 \mathrm{~ms}$ to use the entire three-second time frame with sequence parameters otherwise equal to the iTRUST-inserted OxFlow module. 
For each subject, $T_{2}$-based $Y_{\mathrm{v}}\left(Y_{\mathrm{v}}-T_{2}\right)$ was derived from TRUST and iTRUST data, and SBO-based $Y_{\mathrm{v}}\left(Y_{\mathrm{v}}-\mathrm{SBO}\right)$ and tCBF from iTRUST and OxFlow data. Differences in parameter values across subjects were compared between techniques - TRUST vs. iTRUST for $Y_{\mathrm{v}}-T_{2}$, OxFlow vs. iTRUST for $Y_{\mathrm{v}}-\mathrm{SBO}$ and tCBF - to determine any potential bias in the interleaved approach relative to the separate techniques. Further, $T_{2}$ values obtained from the iTRUST data were recalculated using only the 0 and $80 \mathrm{~ms}$ eTE image pairs to determine any bias caused by using fewer eTEs. $T_{2}$ fitting with two eTEs has previously been demonstrated at $7 \mathrm{~T}$ field strength (Krishnamurthy et al., 2014), where the short $T_{2}$ value of blood precludes the use of longer $T_{2}$ preparations.

\section{Simulations}

The use of fewer eTE images pairs was further explored by simulating TRUST difference signals with a blood $T_{2}$ value of $72 \mathrm{~ms}$, corresponding to typical physiologic values of $Y_{\mathrm{v}}=$ $65 \% \mathrm{HbO}_{2}$ and $\mathrm{Hct}=0.40$, with noise added corresponding to the typically observed SNR range of our acquired TRUST data $(\mathrm{SNR}=20-80)$. This SNR range is similar to that reported in previous studies (Xu et al., 2012b). Exponential fitting was performed and $Y_{\mathrm{V}}$ values were determined from the published calibration curve (Lu et al., 2012) using all four $(0,40,80,160 \mathrm{~ms})$, three $(0,40$, and $80 \mathrm{~ms})$, or two $(0$ and $80 \mathrm{~ms})$ eTEs. Root-mean-square error (RMSE) relative to the true $Y_{\mathrm{v}}$ of $65 \% \mathrm{HbO}_{2}$ was quantified as a function of SNR and number of eTEs used.

\section{Apnea study}

To evaluate the sensitivity of the iTRUST technique to detect dynamic changes inflow, $Y_{\mathrm{v}^{-}}$ SBO, and $Y_{\mathrm{v}}-T_{2}$, a breath-hold challenge was conducted in one healthy subject (age 28 years, male). iTRUST was run with two eTEs ( 0 and $80 \mathrm{~ms}$ ) during a paradigm consisting of two minutes baseline, one-minute breath hold after inhalation, and two minutes recovery. $Y_{\mathrm{v}}$-SBO and tCBF were quantified every three seconds. $Y_{\mathrm{v}}-T_{2}$ values were quantified with sliding-window reconstruction using all adjacent difference image pairs, yielding six-second temporal resolution from 12-second data windows. The mean and standard deviation of the difference between time matched $Y_{\mathrm{v}}-T_{2}$ and $Y_{\mathrm{v}}-\mathrm{SBO}$ values was quantified across all time points, and compared by paired two-sample Student's $t$-tests.

\section{Hypercapnia study}

In 10 subjects, iTRUST comprising only two eTEs ( 0 and $80 \mathrm{~ms}$ ) was applied during a hypercapnia paradigm to determine whether differences exist in the $\mathrm{CMRO}_{2}$ as determined via $T_{2}$ - versus susceptometry-based quantification of $Y_{\mathrm{v}}$. A two-way non-rebreathing Tvalve (2700 Series, Hans Rudolph, Inc., Kansas City, MO, USA) was used to deliver 5\% $\mathrm{CO}_{2}$ in room air for five minutes via a $100 \mathrm{~L}$ Douglas bag. Room air was delivered five minutes before and after hypercapnia, and MRI data were collected continuously for the entire 15 minutes. $Y_{\mathrm{a}}$ and heart rate (HR) were monitored with pulse oximetry, and end-tidal $\mathrm{CO}_{2}\left(\mathrm{EtCO}_{2}\right)$ and respiratory rate (RR) with capnography (Expression, Invivo Research Inc., Orlando, FL, USA).

tCBF, $Y_{\mathrm{v}}-\mathrm{SBO}$, and $\mathrm{SBO}$-based $\mathrm{CMRO}_{2}\left(\mathrm{CMRO}_{2}-\mathrm{SBO}\right)$ parameter values were determined from the OxFlow data at three-second temporal resolution, and $Y_{\mathrm{a}}$ values were sampled at 
three-second intervals to match the MRI data. $Y_{\mathrm{v}}-T_{2}$ values were quantified every six seconds from the EPI data with 12-second sliding-window reconstruction. tCBF and $Y_{\mathrm{a}}$ values were interpolated to the corresponding $Y_{\mathrm{v}}-T_{2}$ time points to determine $T_{2}$-based $\mathrm{CMRO}_{2}\left(\mathrm{CMRO}_{2}-T_{2}\right)$ values every six seconds. For each parameter, means and standard deviations were quantified across the baseline (0-5 minutes) and steady-state hypercapnia (7.5-10 minutes) periods, and used to determine percent changes in response to hypercapnia. Changes in $\mathrm{CMRO}_{2}-\mathrm{T}_{2}$ and $\mathrm{CMRO}_{2}$-SBO in response to hypercapnia were evaluated with one-sample Student's $t$-tests.

\section{Image analysis}

All image reconstructions were performed with in-house-written Matlab (Mathworks, Natick, MA) scripts. BRISK-sampled raw OxFlow data, whether acquired alone or as part of an iTRUST sequence, were first reordered to create full $\mathrm{k}$-space images corresponding to each three-second time point. To determine tCBF, the phase difference between positive gradient-moment flow-encoded and flow-compensated images acquired at $\mathrm{TE}_{1}$ were used to generate velocity maps, and SSSBF was obtained by integrating velocity across the vessel cross-sectional area. Data from the two-slice interleaved PC-MRI sequence used to determine the SSSBF:tCBF ratio were processed analogously. This ratio was then used to upscale the dynamically acquired SSSBF data to determine tCBF.

For $Y_{\mathrm{v}}$-SBO determination, a raw phase difference map was generated from images acquired at $\mathrm{TE}_{1}$ and $\mathrm{TE}_{2}$ of the flow-compensated OxFlow interleave. Low spatial frequency bulk susceptibility effects were removed via second-order polynomial fitting of the induced field in the surrounding brain tissue (Langham et al., 2009). The average phase difference, $\Delta \varphi$, was determined between pixels entirely within the SSS (i.e., without any tissue partial voluming) and pixels in a reference region of brain tissue surrounding the SSS approximately one vessel-radius in width and located one vessel-radius from the SSS border, allowing determination of $Y_{\mathrm{v}}$-SBO from Eqs. (2)-(4).

TRUST or iTRUST EPI data for $T_{2}$-determiation were first reconstructed and corrected for $\mathrm{N} / 2$ ghosting. Difference images were produced for each eTE via tag-control subtraction. As previously described ( $\mathrm{Lu}$ and $\mathrm{Ge}, 2008$ ), the four brightest pixels in the SSS were selected for $T_{2}$ fitting, using a weighted least-squares fit calculated by the Matlab function lscov.m.

\section{Results}

Across the three subjects scanned at baseline, quantified $Y_{\mathrm{v}}$ and tCBF values were consistent with previous reports (Xu et al., 2009; Jain et al., 2010), and mean absolute bias between TRUST and iTRUST $Y_{\mathrm{v}}-T_{2}$ (Fig. 2A) and between OxFlow and iTRUST $Y_{\mathrm{v}}$-SBO (Fig. 2B) and tCBF (Fig. 2C) values were small. These values likely represent an upper bound on any true bias, as they also include contributions from measurement noise and true physiologic variation over the scan duration. Standard deviations of the parameter values varied across subjects, but were similar between techniques, suggesting precision of the combined iTRUST sequence to be comparable to the separate TRUST and OxFlow sequences. 
In Fig. 3, iTRUST $Y_{\mathrm{v}}-T_{2}$ values are shown based on $T_{2}$ fitting using all four $(0,40,80$, and $160 \mathrm{~ms})$ or just two (0 and $80 \mathrm{~ms})$ eTE difference signals. The mean \pm SD difference between the two sets of values was small at $0.2 \pm 1.8 \% \mathrm{HbO}_{2}(P=0.65)$. The $95 \%$ confidence interval for the linear least-squares regression line includes the line of identity, further indicating that no significant bias is introduced by using two instead of four eTEs. $Y_{\mathrm{v}}-T_{2}$ variability was slightly larger when using two versus four eTEs (subject-averaged SDs of 2.6 and $1.6 \% \mathrm{HbO}_{2}$, respectively). However, this difference is largely eliminated if RMSEs are scan-time normalized, that is, after multiplying by $\sqrt{\text { number eTEs }}$ used for fitting. These data support the use of two eTEs in subsequent iTRUST experiments.

Fig. 4 shows RMSEs for $Y_{\mathrm{v}}-T_{2}$ values across the typical TRUST SNR range, both absolute (Fig. 4A) and scan-time normalized (Fig. 4B). Even before normalization, three and two eTEs result in less error than four eTEs. Normalized for scan time, both three and two eTEs perform significantly better than four eTEs, with $\approx 30 \%-45 \%$ reduction in RMSE across the SNR range.

iTRUST with two eTEs was evaluated in response to breath-hold apnea to test the ability of the technique to detect dynamic physiologic processes. A time-course plot of the extracted parameter values (Fig. 5) demonstrates the expected apneic response of increased $Y_{\mathrm{V}}$ and tCBF (Rodgers et al., 2013a). $Y_{\mathrm{v}}-\mathrm{SBO}$ and $Y_{\mathrm{v}}-T_{2}$ values match closely, with $Y_{\mathrm{v}}$-SBO values higher by an average of $1.5 \pm 3.0 \% \mathrm{HbO}_{2}(P<0.01)$.

All 10 subjects were able to successfully complete the hypercapnia paradigm. Average brain volume, Hct, and SSS angle $(\theta)$ were $1468 \pm 77 \mathrm{~mL}, 0.43 \pm 0.04$, and $15.2^{\circ} \pm 5.0^{\circ}$, respectively. On average, the SSSBF:tCBF ratio was $0.48 \pm 0.03$, in line with previous studies (Rodgers et al., 2013a). Subject-averaged time-course plots of physiologic parameters measured via pulse oximetry $\left(Y_{\mathrm{a}}, \mathrm{HR}\right)$ and capnography $\left(\mathrm{EtCO}_{2}, \mathrm{RR}\right)$ are displayed in Fig. 6.

Fig. 7 displays a representative subject time-course plot of all MRI-derived parameters (and $\left.Y_{\mathrm{a}}\right)$ in absolute physiologic units (Fig. 7A), subject-averaged plots of both absolute parameter values (Fig. 7B) and baseline-normalized parameter values (Fig. 7C), and a scatter plot comparing $Y_{\mathrm{v}}-\mathrm{SBO}$ and $Y_{\mathrm{v}}-T_{2}$ values across all subjects and time points (Fig. 7D). Parameter values were observed to reach a steady state after approximately 2.5 minutes of hypercapnia. Average baseline ( $0-5$ minutes) and steady-state hypercapnia (7.5-10 minutes) values are displayed in Table $1 . \mathrm{EtCO}_{2}, \mathrm{tCBF}, Y_{\mathrm{v}}-\mathrm{SBO}$, and $Y_{\mathrm{v}}-T_{2}$ all increased significantly in response to hypercapnia $(P<0.0001)$. Subject-averaged cerebrovascular reactivity was $4.6 \pm 0.9 \% \mathrm{tCBF} / \mathrm{mm} \mathrm{Hg} \mathrm{EtCO}$, in line with previous results (Chen and Pike, 2010; Jain et al., 2011).

$Y_{\mathrm{v}}-T_{2}$, although lower than $Y_{\mathrm{v}}-\mathrm{SBO}$ at baseline, increased more during hypercapnia. As a result, in response to hypercapnia $\mathrm{CMRO}_{2}$-SBO did not change significantly $(3.3 \pm 9.7 \%, P$ $=0.31)$, whereas $\mathrm{CMRO}_{2}-T_{2}$ decreased substantially $(-14.6 \pm 3.6 \%, P<0.0001)$.

Following cessation of apnea, $\mathrm{tCBF}$ and $Y_{\mathrm{v}}$ undershot before gradually returning to baseline. $\mathrm{CMRO}_{2}$ values during the end-recovery period (12.5-15 minutes) were not significantly 
different from baseline values $\left(P=0.36\right.$ and $P=0.33$ for $\mathrm{CMRO}_{2}-\mathrm{SBO}$ and $\mathrm{CMRO}_{2}-T_{2}$, respectively).

\section{Discussion}

\section{Validation of iTRUST}

Because changes inflow and $Y_{\mathrm{v}}$ tend to oppose each other both at baseline and in response to stimuli, it is critical to measure these two quantities simultaneously to most accurately determine $\mathrm{CMRO}_{2}$. This is especially important during physiologic stimuli, where temporal mismatch between $Y_{\mathrm{v}}$ and flow quantification could lead to significant errors. iTRUST makes such simultaneous measurement, previously achievable only with susceptometrybased $\mathrm{CMRO}_{2}$ approaches, possible for $T_{2}$-based $\mathrm{CMRO}_{2}$ quantification as well.

Combination of the TRUST and OxFlow techniques in iTRUST did not significantly impact the accuracy or precision of the quantified parameters (Fig. 2). This is expected, as the OxFlow and $T_{2}$-quantificaiton portions of the pulse sequence are separated in such a way that they should not affect one another's spin histories. While less time is available for OxFlow measurement in iTRUST than OxFlow alone for a given temporal resolution (1420 $\mathrm{ms}$ versus $3000 \mathrm{~ms}$ in this study), this did not appear to impact the precision of the OxFlow data as evidenced by similar standard deviations for iTRUST and OxFlow derived parameters (Fig. 2B and C).

\section{$\mathrm{T}_{2}$-based $\mathrm{CMRO}_{2}$ temporal resolution}

Previous implementations of TRUST had a temporal resolution for $\mathrm{CMRO}_{2}$ quantification of several minutes (Liu et al., 2013), compared to as little as three seconds for OxFlow (Rodgers et al., 2013a). This is partially due to the usual acquisition of three TRUST averages (requiring $3 \times 24=72$ seconds) and measurement of each arterial inflow vessel with a separate 30 seconds PC-MRI measurement, which has been demonstrated to produce accurate and reproducible $\mathrm{CMRO}_{2}$ measurements (Liu et al., 2013). While this approach is optimal when a single $\mathrm{CMRO}_{2}$ measure of baseline physiology is the objective, it does not allow for quantification of dynamic changes in $T_{2}$-based $Y_{\mathrm{v}} / \mathrm{CMRO}_{2}$.

iTRUST improves $\mathrm{CMRO}_{2}-T_{2}$ temporal resolution to as little as six seconds via insertion of flow quantification within the $T_{1}$ recovery period and use of two eTEs with sliding window reconstruction. These modifications may also improve measurement precision. For instance, rapid measurement of SSSBF is achieved more easily than quantification of tCBF in the neck arteries, due to the sagittal sinus' larger size, less pulsatile flow, and fixed position in the scanner even during swallowing, breath hold, or gas mixture breathing manipulations that can complicate flow quantification in the neck arteries. Upscaling this dynamically acquired SSSBF to tCBF only requires a single high-quality PC-MRI acquisition before or after accelerated SSSBF-only measurement, since the SSSBF:tCBF ratio has ben observed to remain fixed in response to blood flow changes (Rodgers et al., 2013a).

Simulation results (Fig. 4) suggest that inclusion of a $160 \mathrm{~ms}$ eTE difference image actually reduces $T_{2}$ estimation precision due to its relatively low SNR. For iTRUST at $3 \mathrm{~T}, T_{2}$ measurement based on two eTEs performs slightly better than three eTEs after 
normalization for scan time differences. This is because an eTE of $80 \mathrm{~ms}$ most closely matches the physiologic $T_{2}$ range (60-100 ms). In vivo measurements at baseline suggest a slightly greater $Y_{\mathrm{v}}$ variation when retrospectively using two vs. four eTEs for $T_{2}$ fitting (SDs of 2.6 versus $1.6 \% \mathrm{HbO}_{2}$, respectively). However, this greater variation likely reflects some degree of sensitivity to true physiologic fluctuations - absent in the simulation data - which is more significantly removed through averaging when using all four eTEs for fitting. One limitation of using only two eTEs is that confidence intervals for the exponential fitting (and therefore $Y_{\mathrm{v}}$ ) cannot be derived based on the regression of the exponential fit.

\section{Hypercapnia study}

Parameter values quantified from the hypercapnia data were in good agreement with previous studies using TRUST or OxFlow independently, both in terms of resting state values (Xu et al., 2009; Jain et al., 2010) and changes in response to hypercapnia (Xu et al., 2011; Jain et al., 2011). Specifically, hypercapnia caused significant reduction in $\mathrm{CMRO}_{2}$ $T_{2}(-14.6 \pm 3.6 \%$, mean $\pm \mathrm{SD}$ ), similar to the original TRUST study (Xu et al., 2011) ($13.4 \pm 8.6 \%$, mean $\pm \mathrm{SD}$, calculated from the reported standard error with $N=14$ ), and also a non-significant change in $\mathrm{CMRO}_{2}-\mathrm{SBO}(3.3 \pm 9.7 \%$, mean $\pm \mathrm{SD})$, similar to the original OxFlow study (Jain et al., 2011). It was suggested (Jain et al., 2011) that the negative hypercapnic response observed with TRUST could have been biased due to flow measurement in the SSS, rather than in the neck arteries as was done with OxFlow. However, the present study used only SSS-based flow quantification, yet achieved results consistent with both previous studies (Xu et al., 2011; Jain et al., 2011). This consistency lends additional support to the use of SSS-based quantification of tCBF, a critical requirement for obtaining high temporal resolution $\mathrm{CMRO}_{2}$ quantification with OxFlow and iTRUST. It also strengthens confidence in the accuracy of the iTRUST technique for $Y_{\mathrm{v}}-T_{2}$ quantification, including use of two eTEs for $T_{2}$ fitting. Most strikingly, it implies that the observed bias between $Y_{\mathrm{v}}-T_{2}$ and $Y_{\mathrm{v}}$-SBO values - both in terms of the baseline offset and relative changes in response to hypercapnia - is not due to random error, differences in experimental protocols, or differences in subject populations, but rather a systematic bias between the techniques.

Average baseline $Y_{\mathrm{v}}-T_{2}$ values were observed to be significantly lower than $Y_{\mathrm{v}}$-SBO values $\left(62.3 \pm 3.1\right.$ vs. $66.7 \pm 5.1 \% \mathrm{HbO}_{2}$, respectively, $\left.P<0.05\right)$, consistent with another recent study (Barhoum et al., 2014). Longer TRUST EPI readouts have been shown to cause a systematic underestimation of $T_{2}$ (and therefore $Y_{\mathrm{v}}$ ), especially at lower SNR (Xu et al., 2012b). This effect was hypothesized to be caused by variations in blood flow, and led the authors to recommend use of a shorter (3 ms) EPI TE via application of parallel imaging. While the present study used a longer TE $(8 \mathrm{~ms})$, the slice-select gradient was first momentcompensated. This should prevent signal differences due to varying degrees of intravoxel dephasing in tag/control images acquired at different blood flow velocities. Furthermore, if a flow velocity-dependent bias did exist, the proposed $Y_{\mathrm{v}}-T_{2}$ underestimation would be expected to get worse at higher $Y_{\mathrm{v}}$ values due to the accompanying CBF and heart rate increase during hypercapnia. In fact, the opposite trend was observed, with $Y_{\mathrm{v}}-T_{2}$ values rising significantly more than $Y_{\mathrm{v}}$-SBO values during the hypercapnic stimulus, regardless of the baseline offset between $Y_{\mathrm{v}}-T_{2}$ and $Y_{\mathrm{v}}-\mathrm{SBO}$. This is illustrated by the subject specific 
regression lines in Fig. 7D, all of which had slopes significantly greater than unity ( $\beta=1.47$ $\pm 0.20, P<0.0001$ for $\mathrm{H}_{0}: \beta=1$ ).

As recently described by Xu et al. (2014), a flow-dependent error in $Y_{\mathrm{V}}-\mathrm{SBO}$ values could potentially arise due to phase accumulation as venous blood travels through an inhomogeneous $\mathrm{B}_{0}$ field. This flow-dependent phase accumulation will increase quadratically with echo time and linearly with the dot product between the flow velocity and the background field gradient. Were the background field gradient direction similar in each subject, it could cause a systematic bias toward flow-dependent over- or under-estimation of $Y_{\mathrm{v}}-\mathrm{SBO}$. However, this effect alone cannot explain the observed bias between $Y_{\mathrm{v}}-T_{2}$ and $Y_{\mathrm{v}^{-}}$ SBO values, as it would predict the bias to increase in magnitude with increasing flow velocity, whereas the observed bias reverses direction between the low flow (baseline) and high flow (hypercapnia) states. Detailed investigation of this potential source of error will be explored in future work by applying a quadratic phase model - the "adaptive-quadratic fit" as described by Xu et al. (2014) - to an SBO sequence with several rephased echoes and longer echo times.

In addition to the aforementioned flow effects, another likely source of the observed discrepancy is an error in the calibration of one or both techniques - that is, the values of the constants in the model equations. However, the susceptibility model (Eq. (2)) is considerably simpler, with only two calibration constants $-\Delta \chi_{\text {do }}$ and $\Delta \chi_{\text {oxy }}-$ defining a linear relationship between measured phase, Hct, and $Y_{\mathrm{v}}$. The values of these constants have been validated theoretically (Spees et al., 2001) and experimentally (Spees et al., 2001; Jain et al., 2012) with excellent agreement. In contrast, TRUST requires calibration of a quadratic equation (Eq. (5)) with six linear coefficients (Lu et al., 2012). This calibration equation is based on a two-compartment exchange model, which may be less appropriate than an alternative diffusion-based model (Gardener et al., 2010). Furthermore, unlike SBO, $T_{2}$-based $Y_{\mathrm{V}}$ quantification has a complex dependence on field strength and pulse sequence parameters (e.g., RF inversion pulse, $t_{\mathrm{CPMG}}$ ).

\section{Applications of iTRUST}

In this work, we were interested in directly comparing $T_{2}$ - and susceptometry-based $Y_{\mathrm{v}} /$ $\mathrm{CMRO}_{2}$ values; however, iTRUST could also be used specifically as a high temporal resolution $T_{2}$-based $\mathrm{CMRO}_{2}$ quantification technique. In this case, a single rather than dualecho PC-MRI sequence module could be used, allowing for reduction in the required viewsharing factor. Such a technique could be applied to $\mathrm{CMRO}_{2}$ quantification in the jugular vein, which is less well suited to SBO because of trachea-induced susceptibility artifacts.

A potential clinical application of iTRUST is the assessment of cerebro-vascular reserve (CVR), the ability of the brain to dynamically increase flow in response to a vasodilatory challenge such as hypercapnia or breath hold apnea. Reduced CVR is strongly correlated with increased stroke risk (Gupta et al., 2012) and associated with lower cognitive performance in subjects with mild cognitive impairment and Alzheimer's disease (Richiardi et al., 2014). While CVR has typically been assessed in terms of blood flow changes only, iTRUST and similar techniques for rapid $\mathrm{CMRO}_{2}$ quantification (Rodgers et al., 2013a) allow multi-parametric assessment of the brain's response to stimuli. Because $\mathrm{CMRO}_{2}$ is a 
more direct reflection of oxygen supply and demand, CVR assessed in terms of $\mathrm{CMRO}_{2}$ may provide a more meaningful index of neurovascular dysfunction than traditional flowbased CVR.

The described approach of inserting a fast imaging sequence within a longitudinal signal recovery period has applications beyond iTRUST. $T_{2}$-relaxation-under-phase-contrast (TRU-PC), which uses phase-contrast rather than tag-control isolation of venous blood (Krishnamurthy et al., 2013), and which can probe vessels as small as $1 \mathrm{~mm}$, contains an equivalent signal waiting period as in TRUST. Addition of flow quantification within TRUPC would provide a means of quantifying oxygen flux rather than simply oxygen saturation in small regional vessels not suitable to SBO. An interleaved approach similar to iTRUST has been used to quantify perfusion, intravascular venous oxygen saturation $\left(Y_{\mathrm{V}}\right)$, and $T_{2}{ }^{*}$ (termed PIVOT) via insertion of a multi-echo GRE within the post-label delay of a pulsed ASL sequence (Englund et al., 2013). The technique allowed simultaneous measurement of all three parameters with two-second temporal resolution during a reactive hyperemia paradigm in the leg. Such combination of perfusion and $Y_{\mathrm{v}}$ quantification may also provide a method for improved BOLD fMRI calibration, as suggested in recent work by Driver et al. (2012).

\section{Conclusions}

We presented a novel technique, iTRUST, for combined susceptometry-and $T_{2}$-based quantification of $\mathrm{CMRO}_{2}$ at high temporal resolution. Simulations and in vivo evaluations demonstrate that iTRUST has comparable precision and accuracy relative to the traditional uncombined methods. In addition, iTRUST provides significantly improved temporal resolution for $T_{2}$-based $\mathrm{CMRO}_{2}$ quantification. In summary, iTRUST is a promising method for dynamic assessment of $\mathrm{CMRO}_{2}$, and offers a unique approach for evaluating and comparing susceptometry- and $T_{2}$-based $\mathrm{CMRO}_{2}$ quantification techniques.

\section{Acknowledgments}

Funding: This work was supported by NIH R21-HD069390, K25-HL111422, T32-EB000814, and R01HL109545.

\section{References}

An HY, Lin WL. Quantitative measurements of cerebral blood oxygen saturation using magnetic resonance imaging. J Cereb Blood Flow Metab. 2000; 20(8):1225-1236. [PubMed: 10950383]

Barhoum S, Rodgers ZB, Langham M, Magland JF, Li C, Wehrli FW. Comparison of MRI methods for measuring whole-brain venous oxygen saturation. Magn Reson Med. 2014

Blockley NP, Griffeth VE, Simon AB, Buxton RB. A review of calibrated blood oxygenation leveldependent (BOLD) methods for the measurement of task-induced changes in brain oxygen metabolism. NMR Biomed. 2013; 26(8):987-1003. [PubMed: 22945365]

Bolar DS, Rosen BR, Sorensen AG, Adalsteinsson E. QUantitative Imaging of eXtraction of oxygen and TIssue consumption (QUIXOTIC) using venular-targeted velocity-selective spin labeling. Magn Reson Med. 2011; 66(6):1550-1562. [PubMed: 21674615]

Borghammer P, Chakravarty M, Jonsdottir KY, Sato N, Matsuda H, Ito K, Arahata Y, Kato T, Gjedde A. Cortical hypometabolism and hypoperfusion in Parkinson's disease is extensive: probably even at early disease stages. Brain Struct Funct. 2010; 214(4):303-317. [PubMed: 20361208] 
Bulte DP, Kelly M, Germuska M, Xie J, Chappell MA, Okell TW, Bright MG, Jezzard P. Quantitative measurement of cerebral physiology using respiratory-calibrated MRI. NeuroImage. 2012; 60(1): 582-591. [PubMed: 22209811]

Chen JJ, Pike GB. Human whole blood T2 relaxometry at 3 Tesla. Magn Reson Med. 2009; 61(2): 249-254. [PubMed: 19165880]

Chen JJ, Pike GB. Global cerebral oxidative metabolism during hypercapnia and hypocapnia in humans: implications for BOLD fMRI. J Cereb Blood Flow Metab. 2010; 30(6):1094-1099. [PubMed: 20372169]

Davis TL, Kwong KK, Weisskoff RM, Rosen BR. Calibrated functional MRI: mapping the dynamics of oxidative metabolism. Proc Natl Acad Sci. 1998; 95(4):1834-1839. [PubMed: 9465103]

Doyle M, Walsh EG, Blackwell GG, Pohost GM. Block regional interpolation scheme for k-space (BRISK): a rapid cardiac imaging technique. Magn Reson Med. 1995; 33(2):163-170. [PubMed: 7707905]

Driver ID, Hall EL, Wharton SJ, Pritchard SE, Francis ST, Gowland PA. Calibrated BOLD using direct measurement of changes in venous oxygenation. NeuroImage. 2012; 63(3):1178-1187. [PubMed: 22971549]

Englund EK, Langham MC, Li C, Rodgers ZB, Floyd TF, Mohler ER, Wehrli FW. Combined measurement of perfusion, venous oxygen saturation, and skeletal muscle $\mathrm{T} 2 *$ during reactive hyperemia in the leg. J Cardiovasc Magn Reson. 2013; 15:70. [PubMed: 23958293]

Fan AP, Benner T, Bolar DS, Rosen BR, Adalsteinsson E. Phase-based regional oxygen metabolism (PROM) using MRI. Magn Reson Med. 2012; 67(3):669-678. [PubMed: 21713981]

Fan AP, Bilgic B, Gagnon L, Witzel T, Bhat H, Rosen BR, Adalsteinsson E. Quantitative oxygenation venography from MRI phase. Magn Reson Med. 2014; 72(1):149-159. [PubMed: 24006229]

Fernandez-Seara MA, Techawiboonwong A, Detre JA, Wehrli FW. MR susceptometry for measuring global brain oxygen extraction. Magn Reson Med. 2006; 55(5):967-973. [PubMed: 16598726]

Gardener AG, Francis ST, Prior M, Peters A, Gowland PA. Dependence of blood R2 relaxivity on CPMG echo-spacing at 2.35 and 7 T. Magn Reson Med. 2010; 64(4):967-974. [PubMed: 20715058]

Gauthier CJ, Desjardins-Crepeau L, Madjar C, Bherer L, Hoge RD. Absolute quantification of resting oxygen metabolism and metabolic reactivity during functional activation using QUO2 MRI. NeuroImage. 2012; 63(3):1353-1363. [PubMed: 22986357]

Ge Y, Zhang Z, Lu H, Tang L, Jaggi H, Herbert J, Babb JS, Rusinek H, Grossman RI. Characterizing brain oxygen metabolism in patients with multiple sclerosis with T2-relaxation-under-spin-tagging MRI. J Cereb Blood Flow Metab. 2012; 32(3):403-412. [PubMed: 22252237]

Golay X, Silvennoinen MJ, Zhou J, Clingman CS, Kauppinen RA, Pekar JJ, van Zij PC. Measurement of tissue oxygen extraction ratios from venous blood $\mathrm{T}(2)$ : increased precision and validation of principle. Magn Reson Med. 2001; 46(2):282-291. [PubMed: 11477631]

Guo J, Wong EC. Venous oxygenation mapping using velocity-selective excitation and arterial nulling. Magn Reson Med. 2012; 68(5):1458-1471. [PubMed: 22294414]

Gupta A, Chazen JL, Hartman M, Delgado D, Anumula N, Shao H, Mazumdar M, Segal AZ, Kamel H, Leifer D, Sanelli PC. Cerebrovascular reserve and stroke risk in patients with carotid stenosis or occlusion: a systematic review and meta-analysis. Stroke. 2012; 43(11):2884-2891. [PubMed: 23091119]

Haacke EM, Lai S, Reichenbach JR, Kuppusamy K, Hoogenraad FGC, Takeichi H, Lin WL. In vivo measurement of blood oxygen saturation using magnetic resonance imaging: a direct validation of the blood oxygen level-dependent concept in functional brain imaging. Hum Brain Mapp. 1997; 5(5):341-346. [PubMed: 20408238]

He X, Yablonskiy DA. Quantitative BOLD: mapping of human cerebral deoxygen-ated blood volume and oxygen extraction fraction: default state. Magn Reson Med. 2007; 57(1):115-126. [PubMed: 17191227]

Herscovitch P, Raichle ME. What is the correct value for the brain-blood partition coefficient for water? J Cereb Blood Flow Metab. 1985; 5(1):65-69. [PubMed: 3871783]

Ishii K, Kitagaki H, Kono M, Mori E. Decreased medial temporal oxygen metabolism in Alzheimer's disease shown by PET. J Nucl Med. 1996; 37(7):1159-1165. [PubMed: 8965188] 
Ito H, Ibaraki M, Kanno I, Fukuda H, Miura S. Changes in cerebral blood flow and cerebral oxygen metabolism during neural activation measured by positron emission tomography: comparison with blood oxygenation level-dependent contrast measured by functional magnetic resonance imaging. J Cereb Blood Flow Metab. 2005; 25(3):371-377. [PubMed: 15660103]

Jain V, Langham MC, Wehrli FW. MRI estimation of global brain oxygen consumption rate. J Cereb Blood Flow Metab. 2010; 30(9):1598-1607. [PubMed: 20407465]

Jain V, Langham MC, Floyd TF, Jain G, Magland JF, Wehrli FW. Rapid magnetic resonance measurement of global cerebral metabolic rate of oxygen consumption in humans during rest and hypercapnia. J Cereb Blood Flow Metab. 2011; 31(7):1504-1512. [PubMed: 21505481]

Jain V, Abdulmalik O, Propert KJ, Wehrli FW. Investigating the magnetic susceptibility properties of fresh human blood for noninvasive oxygen saturation quantification. Magn Reson Med. 2012; 68(3):863-867. [PubMed: 22162033]

Jain V, Magland J, Langham M, Wehrli FW. High temporal resolution in vivo blood oximetry via projection-based T(2) measurement. Magn Reson Med. 2014; 70:785-790. [PubMed: 23081759]

Kety SS, Schmidt CF. The effects of altered arterial tensions of carbon dioxide and oxygen on cerebral blood flow and cerebral oxygen consumption of normal young men. J Clin Investig. 1948; 27(4): 484-492. [PubMed: 16695569]

Kim SG, Ogawa S. Biophysical and physiological origins of blood oxygenation level-dependent fMRI signals. J Cereb Blood Flow Metab. 2012; 32(7):1188-1206. [PubMed: 22395207]

Krishnamurthy LC, Liu P, Ge Y, Lu H. Vessel-specific quantification of blood oxygenation with Trelaxation-under-phase-contrast MRI. Magn Reson Med. 2013; 71:978-989. [PubMed: 23568830]

Krishnamurthy LC, Liu P, Xu F, Uh J, Dimitrov I, Lu H. Dependence of blood T(2) on oxygenation at 7 T: in vitro calibration and in vivo application. Magn Reson Med. 2014; 71(6):2035-2042. [PubMed: 23843129]

Langham MC, Magland JF, Floyd TF, Wehrli FW. Retrospective correction for induced magnetic field inhomogeneity in measurements of large-vessel hemoglobin oxygen saturation by MR susceptometry. Magn Reson Med. 2009; 61(3):626-633. [PubMed: 19107914]

Li C, Langham MC, Epstein CL, Magland JF, Wu J, Gee J, Wehrli FW. Accuracy of the cylinder approximation for susceptometric measurement of intravascular oxygen saturation. Magn Reson Med. 2012; 67(3):808-813. [PubMed: 21858859]

Liu P, Xu F, Lu H. Test-retest reproducibility of a rapid method to measure brain oxygen metabolism. Magn Reson Med. 2013; 69(3):675-681. [PubMed: 22517498]

Liu P, Huang H, Rollins N, Chalak LF, Jeon T, Halovanic C, Lu H. Quantitative assessment of global cerebral metabolic rate of oxygen $\left(\mathrm{CMRO}_{2}\right)$ in neonates using MRI. NMR Biomed. 2014a; $27(3)$ : 332-340. [PubMed: 24399806]

Liu J, Zhu YS, Khan MA, Brunk E, Martin-Cook K, Weiner MF, Cullum CM, Lu H, Levine BD, Diaz-Arrastia R, Zhang R. Global brain hypoperfusion and oxygenation in amnestic mild cognitive impairment. Alzheimers Dement. 2014b; 10(2):162-170. [PubMed: 23871763]

Lu H, Ge Y. Quantitative evaluation of oxygenation in venous vessels using T2-relaxation-under-spintagging MRI. Magn Reson Med. 2008; 60(2):357-363. [PubMed: 18666116]

Lu H, Xu F, Grgac K, Liu P, Qin Q, van Zijl P. Calibration and validation of TRUST MRI for the estimation of cerebral blood oxygenation. Magn Reson Med. 2012; 67(1):42-49. [PubMed: 21590721]

Magland, J.; Wehrli, FW. Pulse sequence programming in a dynamic visual environment. Proceedings of the 14th Annual Meeting of ISMRM; Seattle, Washington, USA. 2006. p. 3032

Matthews PM, Honey GD, Bullmore ET. Applications of fMRI in translational medicine and clinical practice. Nat Rev Neurosci. 2006; 7(9):732-744. [PubMed: 16924262]

Mugler JP, Brookeman JR. Three-dimensional magnetization-prepared rapid gradient-echo imaging (3D MP RAGE). Magn Reson Med. 1990; 15(1):152-157. [PubMed: 2374495]

Peng SL, Dumas JA, Park DC, Liu P, Filbey FM, McAdams CJ, Pinkham AE, Adinoff B, Zhang R, Lu H. Age-related increase of resting metabolic rate in the human brain. NeuroImage. 2014; 98:176183. [PubMed: 24814209] 
Qin Q, Grgac K, van Zijl PC. Determination of whole-brain oxygen extraction fractions by fast measurement of blood T(2) in the jugular vein. Magn Reson Med. 2011; 65(2):471-479. [PubMed: 21264936]

Richiardi J, Monsch AU, Haas T, Barkhof F, Van de Ville D, Radu EW, Kressig RW, Haller S. Altered cerebrovascular reactivity velocity in mild cognitive impairment and Alzheimer's disease. Neurobiol Aging. 2014

Rodgers ZB, Jain V, Englund EK, Langham MC, Wehrli FW. High temporal resolution MRI quantification of global cerebral metabolic rate of oxygen consumption in response to apneic challenge. J Cereb Blood Flow Metab. 2013a; 33(10):1514-1522. [PubMed: 23838827]

Rodgers, ZB.; Kini, L.; Langham, M.; Magland, J.; Wehrli, FW. High temporal resolution, simultaneous quantification of intravascular blood flow and oxygen saturation with BRISK kspace sampling. Proceedings of 21st Annual Meeting of ISMRM; Salt Lake City, Utah, USA. 2013b. p. 1351

Rolfe DF, Brown GC. Cellular energy utilization and molecular origin of standard metabolic rate in mammals. Physiol Rev. 1997; 77(3):731-758. [PubMed: 9234964]

Smith SM. Fast robust automated brain extraction. Hum Brain Mapp. 2002; 17(3):143-155. [PubMed: 12391568]

Spees WM, Yablonskiy DA, Oswood MC, Ackerman JJ. Water proton MR properties of human blood at 1.5 Tesla: magnetic susceptibility, $\mathrm{T}(1), \mathrm{T}(2), \mathrm{T}^{*}(2)$, and non-Lorentzian signal behavior. Magn Reson Med. 2001; 45(4):533-542. [PubMed: 11283978]

Van Vaals JJ, Brummer ME, Thomas Dixon W, Tuithof HH, Engels H, Nelson RC, Gerety BM, Chezmar JL, Den Boer JA. "Keyhole" method for accelerating imaging of contrast agent uptake. J Magn Reson Imaging. 1993; 3(4):671-675. [PubMed: 8347963]

Wright GA, Hu BS, Macovski A. Estimating oxygen saturation of blood in vivo with MR imaging at 1.5 T. J Magn Reson Imaging. 1991; 1(3):275-283. [PubMed: 1802140]

Xu F, Ge Y, Lu H. Noninvasive quantification of whole-brain cerebral metabolic rate of oxygen $\left(\mathrm{CMRO}_{2}\right)$ by MRI. Magn Reson Med. 2009; 62(1):141-148. [PubMed: 19353674]

Xu F, Uh J, Brier MR, Hart J Jr, Yezhuvath US, Gu H, Yang Y, Lu H. The influence of carbon dioxide on brain activity and metabolism in conscious humans. J Cereb Blood Flow Metab. 2011; 31(1): 58-67. [PubMed: 20842164]

Xu F, Liu P, Pascual JM, Xiao G, Lu H. Effect of hypoxia and hyperoxia on cerebral blood flow, blood oxygenation, and oxidative metabolism. J Cereb Blood Flow Metab. 2012a; 32(10):19091918. [PubMed: 22739621]

Xu F, Uh J, Liu P, Lu H. On improving the speed and reliability of T2-relaxation-under-spin-tagging (TRUST) MRI. Magn Reson Med. 2012b; 68(1):198-204. [PubMed: 22127845]

Xu B, Liu T, Spincemaille P, Prince M, Wang Y. Flow compensated quantitative susceptibility mapping for venous oxygenation imaging. Magn Reson Med. 2014; 72(2):438-445. [PubMed: 24006187]

Yablonskiy DA. Cerebral metabolic rate in hypercapnia: controversy continues. J Cereb Blood Flow Metab. 2011; 31(7):1502-1503. [PubMed: 21427732]

\section{Abbreviations}

$\begin{array}{ll}\text { ASL } & \text { arterial spin labeling } \\ \text { AVO }_{2} D & \text { arteriovenous oxygen saturation difference } \\ \text { BOLD } & \text { blood-oxygen-level dependent } \\ \text { CBF } & \text { cerebral blood flow } \\ \text { CMRO }_{2} & \text { cerebral metabolic rate of oxygen } \\ \text { CMRO }_{2} \text {-SBO } & \text { SBO-based } \mathrm{CMRO}_{2}\end{array}$




\begin{tabular}{|c|c|}
\hline $\mathrm{CMRO}_{2}-T_{2}$ & $T_{2}$-based $\mathrm{CMRO}_{2}$ \\
\hline CSF & cerebrospinal fluid \\
\hline $\mathrm{EtCO}_{2}$ & end-tidal $\mathrm{CO}_{2}$ \\
\hline eTE & effective echo time \\
\hline fMRI & functional MRI \\
\hline GRE & gradient-recalled echo \\
\hline$\% \mathrm{HbO}_{2}$ & percent hemoglobin oxygen saturation \\
\hline Het & hematocrit \\
\hline HR & heart rate \\
\hline iTRUST & interleaved TRUST \\
\hline PC-MRI & phase-contrast MRI \\
\hline PET & positron emission tomography \\
\hline RMSE & root-mean-square error \\
\hline $\mathbf{R R}$ & respiratory rate \\
\hline SBO & susceptometry-based oximetry \\
\hline SNR & signal-to-noise ratio \\
\hline SSS & superior sagittal sinus \\
\hline SSSBF & SSS blood flow \\
\hline tCBF & total CBF \\
\hline$t_{\mathrm{CPMG}}$ & CMPG spacing \\
\hline TRU-PC & $T_{2}$-relaxation-under-phase-contrast \\
\hline TRUST & $T_{2}$-relaxation-under-spin-tagging \\
\hline$Y_{\mathbf{a}}$ & arterial oxygen saturation \\
\hline$Y_{\mathbf{v}}$ & venous oxygen saturation \\
\hline$Y_{\mathrm{v}}-\mathrm{SBO}$ & SBO-based $Y_{\mathrm{V}}$ \\
\hline$Y_{\mathrm{v}}-T_{2}$ & $T_{2}$-based $Y_{\mathrm{v}}$ \\
\hline
\end{tabular}




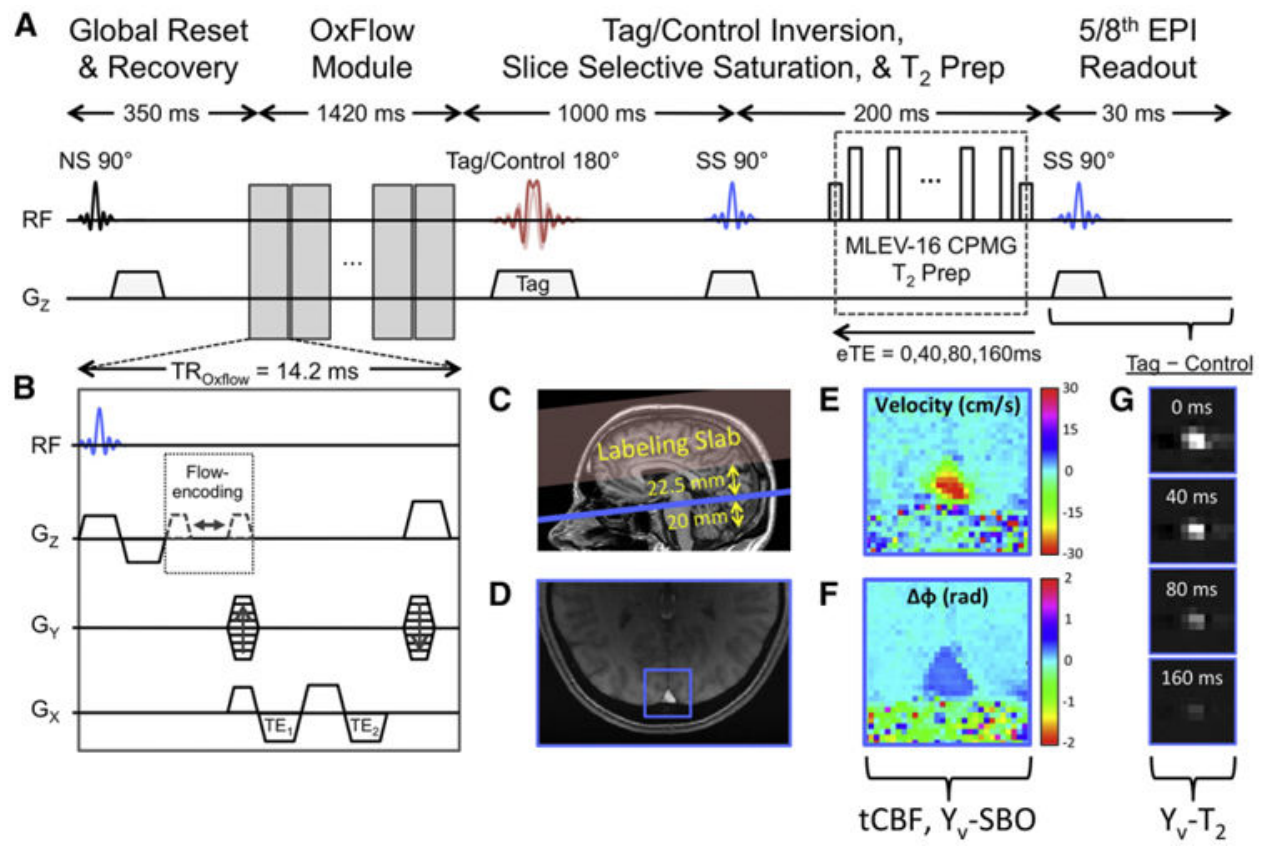

Fig. 1.

iTRUST pulse sequence and example images. (A) TRUST sequence diagram with (B) inset depicting the OxFlow module inserted within the $T_{1}$ recovery period of the TRUST sequence. (C) Sagittal scout image indicating the relative positions of the labeling slab (red) and imaging slice (blue). (D) Magnitude image with square region of interest indicating the position of the SSS. (E) Velocity map and (F) phase difference map of the SSS ROI from (D). (G) TRUST difference images for each eTE. Note that the spin histories of the OxFlow module and TRUST sequence should not interact as they are isolated by the global spin reset and the spatial separation of the imaging slice and labeling slab. 

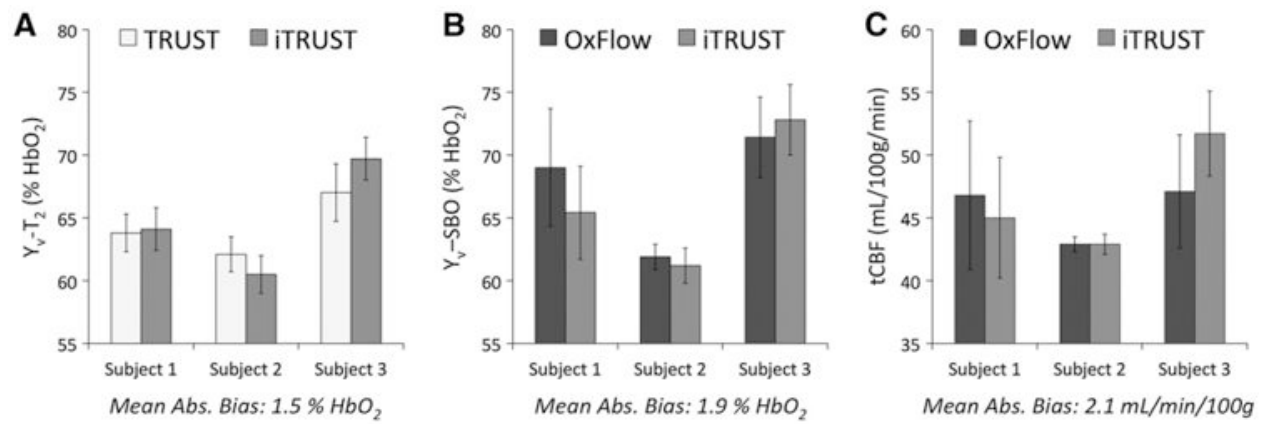

Fig. 2.

TRUST, OxFlow, and iTRUST parameter values acquired sequentially for four minutes each in three resting subjects. (A) TRUST vs. iTRUST $Y_{\mathrm{v}}-T_{2}$ values. (B) OxFlow vs.

iTRUST $Y_{\mathrm{v}}$-SBO values. (C) OxFlow vs. iTRUST tCBF values. Mean absolute bias is the absolute value of the bias between techniques, averaged across subjects. Error bars indicate \pm 1 SD across the $N=10$ (A) or $N=80$ (B and C) data points collected in each four-minute acquisition. 


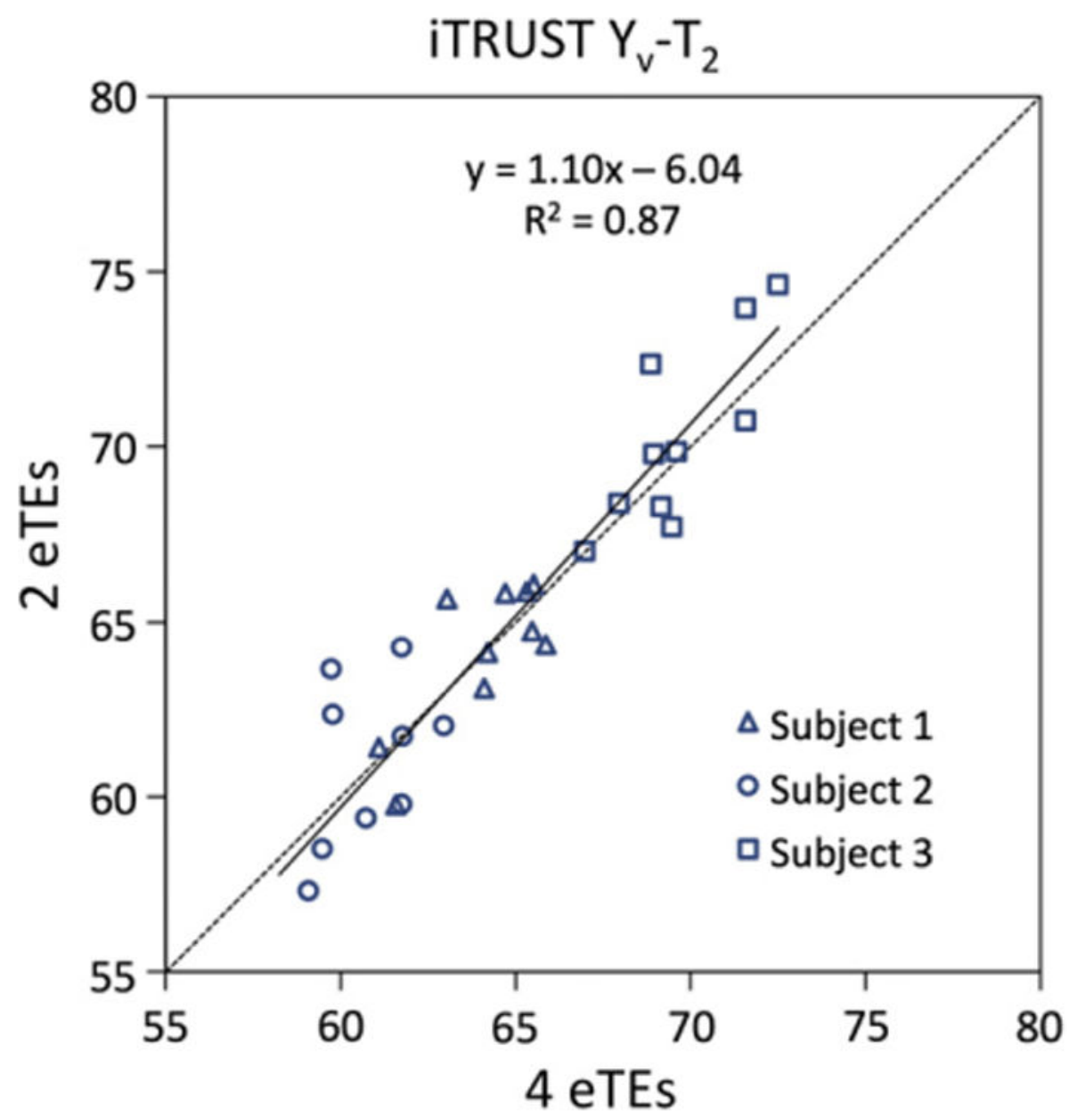

Fig. 3.

Scatter plot of iTRUST $Y_{\mathrm{v}}-T_{2}$ values fitted using all four $(0,40,80$, and $160 \mathrm{~ms})$ vs. only two $(0$ and $80 \mathrm{~ms})$ eTEs from the same data. The 30 data points represent 10 repeat measures from each of three subjects. The linear least-squares regression line for all data points (solid line) is shown along side the line of identity (dotted line). $95 \%$ confidence intervals for the slope $[0.93,1.25]$ and intercept $[-16.4,4.3]$ of the linear fit contain 1 and 0 , respectively, indicating no statistically significant bias between the four and two eTE $Y_{\mathrm{v}}-T_{2}$ values. 

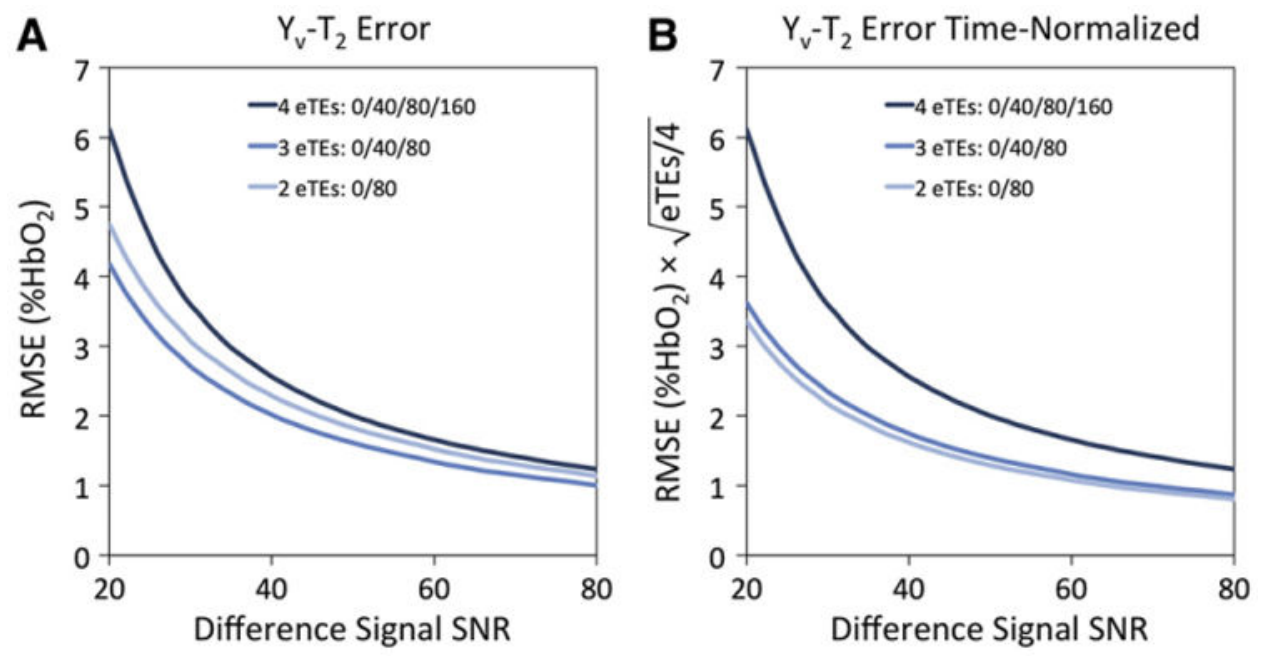

Fig. 4.

Simulation of expected $Y_{\mathrm{v}}-T_{2}$ error vs. the number of eTEs used for $T_{2}$ fitting. (A) Rootmean-square error (RMSE) in $Y_{\mathrm{v}}-T_{2}$ vs. TRUST difference signal SNR for four, three, or two eTEs. (B) RMSE normalized to acquisition time. Simulations were performed for $N=$ 1000 virtual images for each SNR value, incremented by an SNR value of 1 . 


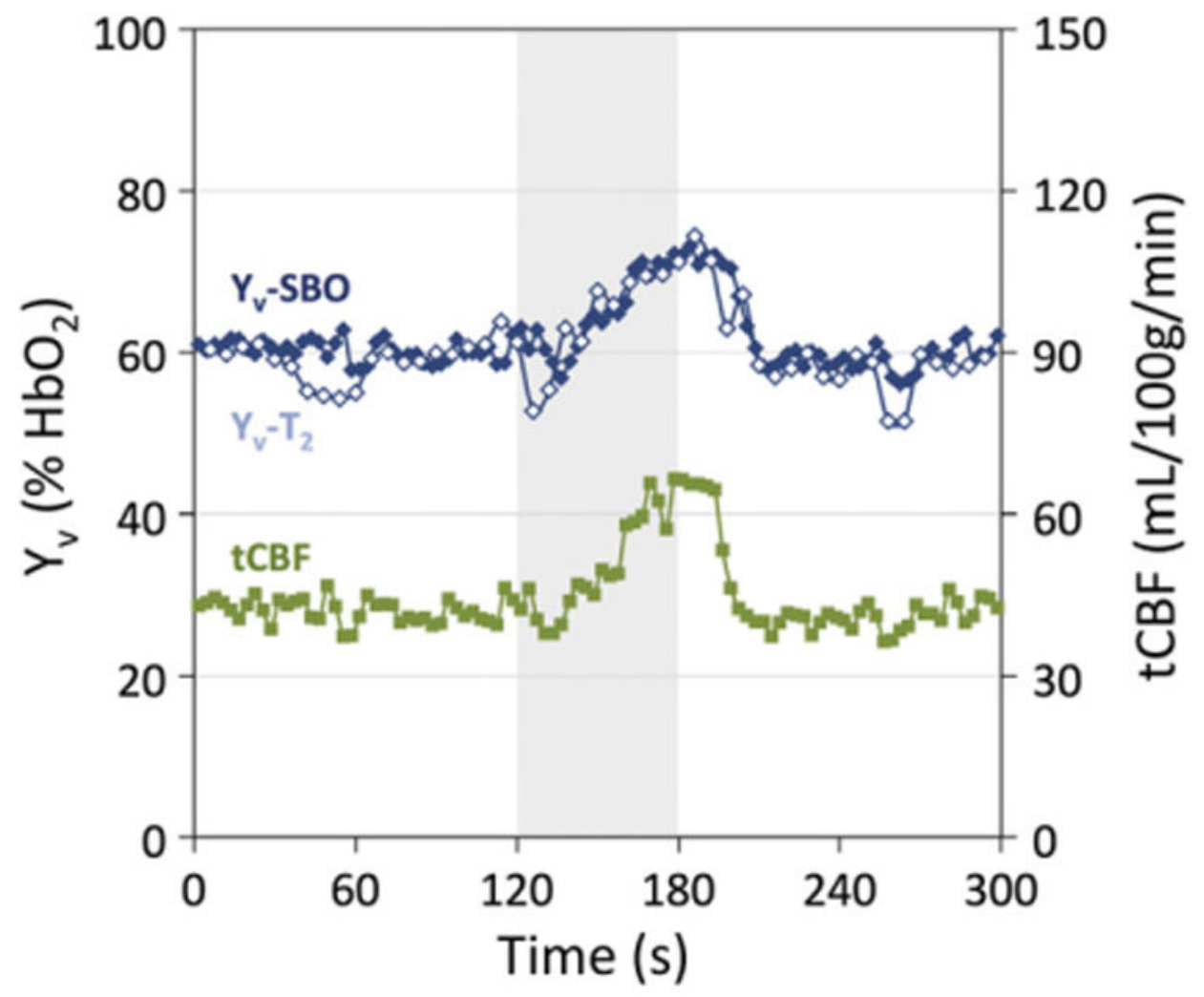

Fig. 5.

iTRUST parameter values in response to a 60 -second breath-hold in a single subject. $T_{2}$ fitting with two eTEs and application of sliding window reconstruction yields $Y_{\mathrm{v}}-T_{2}$ temporal resolution of six seconds. $Y_{\mathrm{v}}$-SBO and tCBF temporal resolution is three seconds. Gray shading indicates the apnea period. 


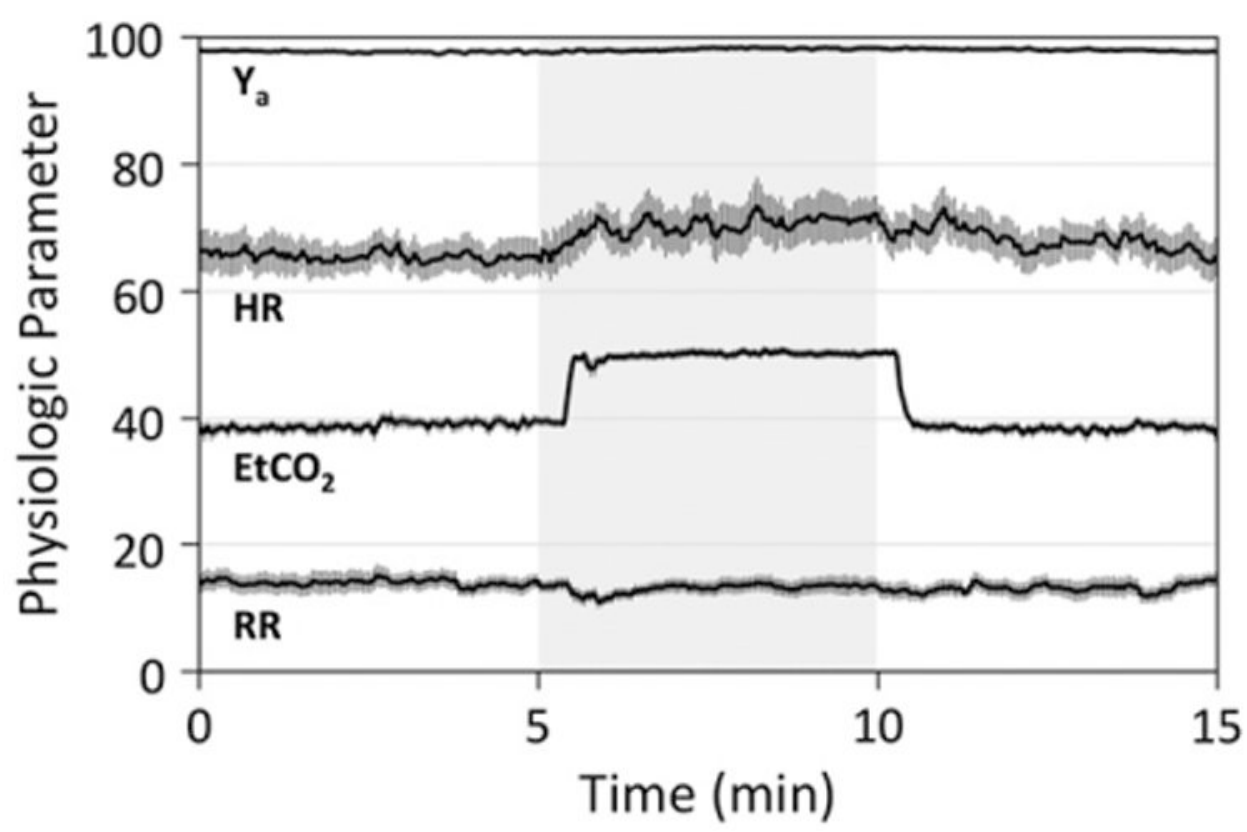

Fig. 6.

Subject-averaged time-course plots of physiologic parameters measured via pulse oximetry $\left(Y_{\mathrm{a}}, \mathrm{HR}\right)$ and capnography $\left(\mathrm{EtCO}_{2}, \mathrm{RR}\right)$. Gray shading indicates the hypercapnia period. Error bars indicate standard errors $(N=10)$. Comparing average baseline $(0-5$ minutes $)$ and steady-state hypercapnia (7.5-10minutes) values across subjects, significant increases were observed in $\mathrm{EtCO}_{2}(P<0.0001), Y_{\mathrm{a}}(P<0.01)$, and HR $(P<0.05)$. RR did not show a significant change $(P=0.64)$. 

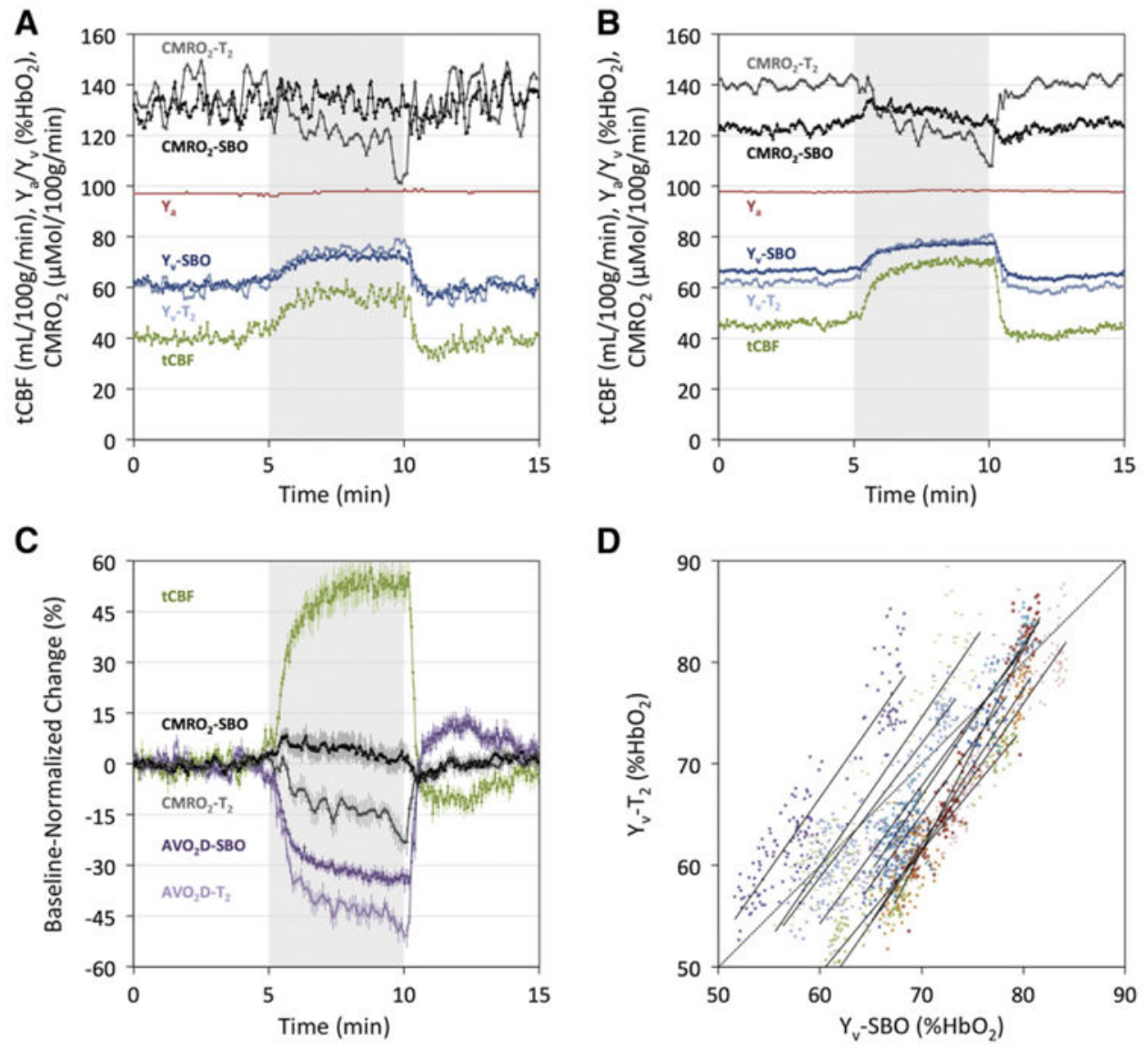

Fig. 7.

iTRUST-derived parameter values inresponse to five minutes of $5 \% \mathrm{CO}_{2}$ gas mixture breathing. (A) Time-course plots of absolute parameter values from (A) a representative subject and (B) averaged across all 10 subjects. (C) Subject-averaged parameter values normalized to average baseline values, with error bars indicating standard errors $(N=10)$ at each time point. The arteriovenous oxygen saturation difference $\left(\mathrm{AVO}_{2} \mathrm{D}\right)$ is equal to $Y_{\mathrm{a}}-$ $Y_{\mathrm{v}}$. In all time-course plots, $\mathrm{tCBF}, Y_{\mathrm{a}}, Y_{\mathrm{v}}-\mathrm{SBO}, \mathrm{AVO}_{2} \mathrm{D}-\mathrm{SBO}$, and $\mathrm{CMRO}_{2}-\mathrm{SBO}$ temporal resolution is three seconds, and $Y_{\mathrm{v}}-T_{2}, \mathrm{AVO}_{2} \mathrm{D}-\mathrm{i}_{2}$, and $\mathrm{CMRO}_{2}-T_{2}$ temporal resolution is six seconds. Gray shading indicates the hypercapnia period. (D) Scatter plot of time-matched $Y_{\mathrm{v}}-\mathrm{SBO}$ and $Y_{\mathrm{v}}-T_{2}$ values across all subjects and time points $(N=1490)$, with different symbols/colors denoting individual subjects. Linear least-squares regression lines are plotted for each subject (solid lines), as well as the line of identity (dotted line). Mean slope and $R^{2}$ values of the regression lines across subjects are $\beta=1.47 \pm 0.20$ and $R^{2}=0.90 \pm 0.02$. 


\section{Table 1}

Summary of hypercapnia paradigm parameter values derived from pulse oximetry, capnography, and iTRUST MRI in 10 subjects.

\begin{tabular}{lrrrr}
\hline Parameter & Baseline (0-5 min) & Hypercapnia (7.5-10 min) & Change (\%) & $P$-value \\
\hline $\mathrm{EtCO}_{2}$ & $38.5(2.9)$ & $50.1(2.1)$ & $30.5(5.5)$ & $<0.0001$ \\
$Y_{\mathrm{a}}$ & $97.7(0.6)$ & $98.3(0.7)$ & $0.6(0.6)$ & $<0.01$ \\
$\mathrm{tCBF}$ & $45.7(6.0)$ & $70.1(11.4)$ & $53.0(12.7)$ & $<0.0001$ \\
$Y_{\mathrm{v}}-\mathrm{SBO}$ & $66.7(5.1)$ & $77.2(4.8)$ & $15.9(2.8)$ & $<0.0001$ \\
$Y_{\mathrm{v}}-T_{2}$ & $62.3(3.1)$ & $78.4(3.5)$ & $25.8(3.7)$ & $<0.0001$ \\
$\mathrm{AVO}_{2} \mathrm{D}-\mathrm{SBO}$ & $31.1(4.8)$ & $21.1(4.4)$ & $-32.5(4.7)$ & $<0.0001$ \\
$\mathrm{AVO}_{2} \mathrm{D}-T_{2}$ & $35.4(2.9)$ & $19.9(3.0)$ & $-43.8(5.9)$ & $<0.0001$ \\
$\mathrm{CMRO}_{2}-\mathrm{SBO}$ & $123.4(18.7)$ & $127.9(25.7)$ & $3.3(9.7)$ & 0.31 \\
$\mathrm{CMRO}_{2}-T_{2}$ & $140.4(9.7)$ & $120.0(9.5)$ & $-14.6(3.6)$ & $<0.0001$ \\
\hline
\end{tabular}

Parentheses indicate the standard deviations of parameter values across subjects. $P$-values are based on one-sample Student's $t$-tests of the percent changes from baseline to hyper-capnia. $\mathrm{AVO}_{2} \mathrm{D}$ is the arteriovenous oxygen difference, equal to $Y_{\mathrm{a}}-Y_{\mathrm{V}}$. 